\title{
Dietary n-3 PUFA May Attenuate Experimental Colitis
}

\author{
Cloé Charpentier, ${ }^{1,2}$ Ronald Chan, ${ }^{3}$ Emmeline Salameh, ${ }^{1}$ Khaly Mbodji, ${ }^{1}$ Aito Ueno, ${ }^{3,4}$ \\ Moïse Coëffier, ${ }^{1,5}$ Charlène Guérin, ${ }^{1}$ Subrata Ghosh $\mathbb{D}^{3},{ }^{3,6}$ Guillaume Savoye, ${ }^{1,2}$ \\ and Rachel Marion-Letellier ${ }^{1}$
}

${ }^{1}$ INSERM UMR 1073, UFR de Médecine-Pharmacie, 22 boulevard Gambetta, 76183 Rouen Cedex, France

${ }^{2}$ Department of Gastroenterology, Rouen University Hospital, 1 rue de Germont, 76031 Rouen Cedex, France

${ }^{3}$ University of Calgary, Gastrointestinal Research Group, Snyder Institute for Chronic Diseases, Calgary, AB, Canada

${ }^{4}$ Center for Advanced IBD Research and Treatment, Kitasato Institute Hospital, Tokyo, Japan

${ }^{5}$ Nutrition Unit, Rouen University Hospital, 1 rue de Germont, 76031 Rouen Cedex, France

${ }^{6}$ Institute of Translational Medicine, University of Birmingham, Birmingham, UK

Correspondence should be addressed to Subrata Ghosh; sughosh@ymail.com

Received 28 July 2017; Revised 13 October 2017; Accepted 31 October 2017; Published 15 February 2018

Academic Editor: Sung-Ling Yeh

Copyright ( 92018 Cloé Charpentier et al. This is an open access article distributed under the Creative Commons Attribution License, which permits unrestricted use, distribution, and reproduction in any medium, provided the original work is properly cited.

Background. Inflammatory bowel diseases (IBD) occurred in genetically predisposed people exposed to environmental triggers. Diet has long been suspected to contribute to the development of IBD. Supplementation with n-3 polyunsaturated fatty acids (PUFA) protects against intestinal inflammation in rodent models while clinical trials showed no benefits. We hypothesized that intervention timing is crucial and dietary fatty acid pattern may influence intestinal environment to modify inflammation genesis. The aim of this study was to evaluate the dietary effect of PUFA composition on intestinal inflammation. Methods. Animals received diet varying in their PUFA composition for four weeks before TNBS-induced colitis. Colon inflammatory markers and gut barrier function parameters were assessed. Inflammatory pathway PCR arrays were determined. Results. n-3 diet significantly decreased colon iNOS, COX-2 expression, IL-6 production, and LTB4 production but tended to decrease colon TNF $\alpha$ production $(P=0.0617)$ compared to control diet. Tight junction protein (claudin-1, occludin) expressions and MUC2 and TFF3 mRNA levels were not different among groups. n-9 diet also decreased colon IL-6 production $(P<0.05)$. Conclusions. Dietary n-3 PUFA influence colitis development by attenuating inflammatory markers. Further research is required to better define dietary advice with a scientific rationale.

\section{Introduction}

Inflammatory bowel diseases (IBD) affects genetically predisposed people exposed to environmental triggers [1]. Amongst environmental factors, dietary habits have long been suspected to contribute to the development of IBD [2]. IBD patients often considered diet as a potential trigger for initiating the disease or causing a relapse [3], and this concept led to exclusion diets especially in children [4].

An increased incidence of IBD has been associated with diets high in animal protein. Indeed, association between dietary pattern (fat/protein) and Crohn's disease (CD) risk was found in a study from Japan [5] while increased consumption of animal protein has been associated with higher IBD risk in a study from France [6]. A systematic review demonstrated that this Western dietary pattern (high fat, high n-6 polyunsaturated fatty acids (PUFA), and high meat) is associated with an increased IBD risk [7]. More recently, a study was conducted in 103 IBD patients using food frequency questionnaire over 1 year and the authors found a positive association between meat intake and disease relapse [8]. Similarly, Western diet had a deleterious impact on gut barrier function and dysbiosis in IBD murine models [9].

While n-3 and n-6 PUFA are essential in human nutrition, a Western diet is characterized by an unbalanced ratio 
of both types of PUFA (n-3/n-6 ratio). Indeed, linoleic acid (LA, n-6 PUFA) consumption has markedly increased (3-fold throughout the 20th century) [10]. Numerous epidemiological studies highlighted the role of dietary intake of monounsaturated fatty acids (MUFA) or PUFA in ulcerative colitis (UC) development. Higher intake of LA is associated with an increased risk of UC [11], while docosahexaenoic acid (DHA) (n-3 PUFA) [12] or oleic acid (n-9 MUFA) $[13,14]$ consumption is beneficial. Ananthakrishnan et al. found that greater fish intake was associated with lower risk of CD [15].

We and others demonstrated an anti-inflammatory effect of $n-3$ polyunsaturated fatty acids in rodent IBD models [16-21] while clinical trials failed [22]. We hypothesized that intervention timing is crucial and dietary fatty acid pattern may influence intestinal environment to modify inflammation genesis [23].

The aim of the study was to investigate the dietary influence of fatty acid composition before the onset of intestinal inflammation by administration of 2,4,6-trinitrobenzene sulfonic acid (TNBS). For this purpose, rats were fed with diets varying in $n-3 / n-6 / n-9$ ratio to reproduce dietary pattern from a pragmatic to a Western diet.

\section{Materials and Methods}

2.1. Animals and Study Design. Young Sprague-Dawley male rats weighing 75-100 g were purchased from Janvier (Le Genest St. Isle, France) and allowed to access food and water ad libitum. After 1 week acclimatization, 50 rats were randomly divided into 5 experimental groups; the control (CTRL) group was fed with control diet and received the vehicle, while colitic groups including TNBS, n-3, n-6, and n-9 groups were fed with control diet, n-3 diet, n- 6 diet, and n-9 diet, respectively, and received TNBS for the colitis induction. Weight changes throughout the study were monitored every day. After 4 weeks of experimental diets (day 28 to day 1 ), the rats underwent 24 hours food deprivation prior to the TNBS or vehicle administration. During the colitis induction (day 0 to 2), rats were provided control diet. The overview of experimental design is illustrated in Figure 1(a).

All animal handling and treatment procedures were performed in accordance with both French national regulations and European Union regulations (Official Journal of the European Community L 358, 18/12/1986) and RML is authorized to use this animal protocol by the French government (Authorization $\mathrm{n}^{\circ} 76-106$ ).

2.2. Diets. Four types of isocaloric and isolipidic experimental diets were prepared with several fatty acid proportions:

(i) The normal diet matched a balanced diet with a $\mathrm{n}-3 / \mathrm{n}-6 / \mathrm{n}-9$ ratio equal to $1: 4: 16$ as a fat ratio recommended by dietary guidelines and described as a well-balanced diet in the literature [24]. The control diet was given to CTRL and TNBS groups. The recommended dietary $n-6 / n-3$ ratio is about 4 in human nutrition which is comparable to the control diet in this study. (ii) $\mathrm{n}-3$ diet had a $\mathrm{n}-3 / \mathrm{n}-6 / \mathrm{n}-9$ ratio equal to $1: 1: 4$. We chose a $n-3 / n-6$ ratio equal to $1: 1$ because this ratio was a target by dietary advice in a Japanese clinical trial for IBD patients [25]. In addition, a n-3/n-6 ratio is commonly used in experimental studies investigating the effect of n-3 therapy [24].

(iii) n-6 diet fitted the Western diet with a n-3/n-6/n-9 ratio at $1: 16: 16$. The dietary $n-6 / n-3$ ratio is about 15 in human Western diets [26], and this ratio is useful to underline the imbalance that characterizes Western diets.

(iv) $n-9$ diet had a similar $n-3 / n-6$ ratio to CTRL diet but is enriched in n-9 MUFA. This n-3/n-9 ratio equal to $1: 24$ is comparable to the ratio observed in people following the Mediterranean diet [26].

Detailed diet composition is shown in Table 1.

2.3. Induction of Colitis. Administration of TNBS (SigmaAldrich Company, Saint-Quentin-Fallavier, France) was employed for colitis induction as previously described [16] in TNBS, n-3, n-6, and n-9 groups (colitic groups). The rats were sacrificed using anesthetic reagents (ketamine and xylazine) at day 2 for further analyses.

2.4. Western Blot. PBS, protease inhibitor cocktail, and phosphatase inhibitor cocktail were purchased from SigmaAldrich (Saint-Quentin-Fallavier, France). The 4-12\% NuPAGE gels and SeeBlue multicolored standard were obtained from Invitrogen (Cergy-Pontoise, France). Frozen colon samples were homogenized in PBS with $0.1 \%$ protease inhibitor cocktail and $1 \%$ phosphatase inhibitor cocktail. Homogenates were centrifuged $\left(12,000 \mathrm{~g}, 15 \mathrm{~min}, 4^{\circ} \mathrm{C}\right)$ and the supernatants were collected. Protein concentration was determined following Bradford's colorimetric method. Aliquots of supernatants containing equal amounts of protein $(30 \mu \mathrm{g})$ were separated on $4-12 \%$ NuPAGE and then transferred to a nitrocellulose membrane (Hybond, GE Healthcare, UK). The mouse monoclonal antibody antiPPAR $\gamma$ (sc-7273), the goat polyclonal anti-COX-2 (sc1747), the mouse anti-iNOS (sc-7271), the rabbit polyclonal anti-HNF-4 (sc-8987), and the HRP-conjugated secondary antibodies were obtained from Santa Cruz Biotechnology (Tebu, Le Perray-en-Yvelines, France). The rabbit anticlaudin-1 and the mouse anti-occludin were, respectively, obtained from Life technology and Invitrogen. After blocking, membranes were incubated with specific primary antibodies at the dilution of $1: 100$ (iNOS), 1:500 (COX-2, HNF-4, and PPAR $\gamma$ ), and $1: 1000$ (claudin-1, occludin. After three washes, membranes were then incubated with the secondary HRP-linked anti-goat IgG (for COX-2), antirabbit IgG (for HNF-4, claudin-1), and anti-mouse IgG (for iNOS, PPAR $\gamma$, and occludin) antibodies. The enhanced chemiluminescence light-detecting kit (GE Healthcare, USA) was utilized for immunodetection. Densitometric data were measured following normalization to the housekeeping protein $(\beta$-actin) by a Scientific Imaging Systems (Image QuantTL, GE Healthcare). 


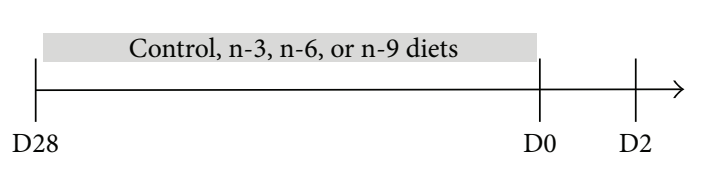

(a)

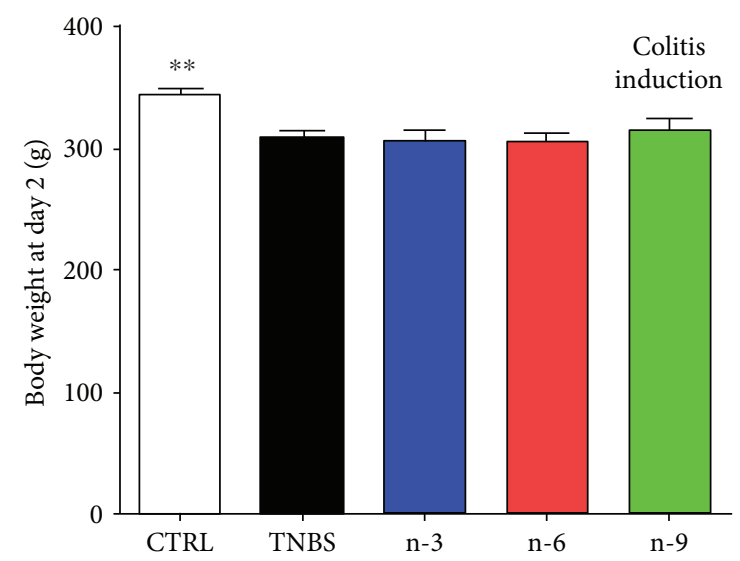

(b)

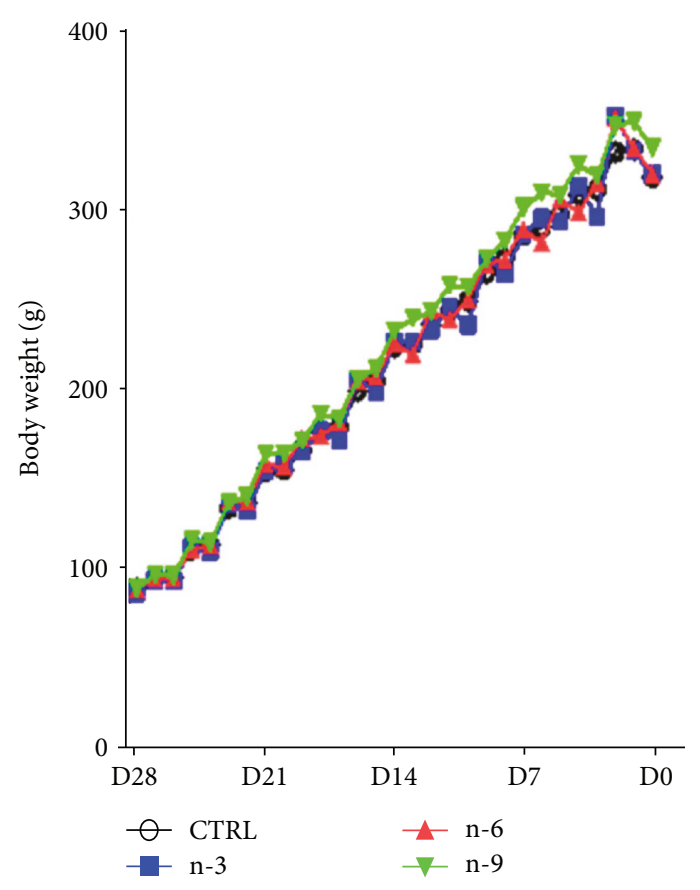

(c)

FIGURE 1: Experimental design and clinical parameters in rats receiving diets varying in their unsaturated fatty acid composition followed by TNBS-induced colitis. (a) Experimental design. Rats received diets varying in their PUFA composition for four weeks before colitis induction at day 0 . Rats were killed at day 2. (b) Body weight at day 2. (c) Body weight follow-up from day 28 to day 2 . $* *$ means $P<0.01$ versus all colitis groups (TNBS, n-3, n-6, and n-9).

TABLE 1: Fatty acid composition of the experimental diets.

\begin{tabular}{lcccc}
\hline & CTRL & n-3 diet & n-6 diet & n-9 diet \\
\hline Total fat (g/1000 g of diet) & 49.7 & 49.4 & 49.7 & 49.8 \\
Saturated fat (g) & 10.2 & 9.9 & 9.3 & 9.6 \\
MUFA (g) & 29.8 & 26.2 & 20.1 & 32.8 \\
PUFA (g) & 9.8 & 13.3 & 20.3 & 7.4 \\
n-6 fatty acids (g) & 7.9 & 7.3 & 19.1 & 6.1 \\
n-3 fatty acids (g) & 1.8 & 6.1 & 1.2 & 1.3 \\
n-9 fatty acids (g) & 29.3 & 25.8 & 19.8 & 32.2 \\
n-3/n-6/n-9 ratio & $1: 4: 16$ & $1: 1: 4$ & $1: 16: 16$ & $1: 4: 24$ \\
\hline
\end{tabular}

2.5. RNA Isolation and Gene Expression Analyses. Colon samples were frozen in liquid nitrogen and stored at $-80^{\circ} \mathrm{C}$ before RNA preparation. Total RNA was isolated from rat colon specimens using a commercial RNA purification kit (SV total RNA isolation kit, Promega, Madison, WI) and mRNA expression of Muc2 (primer sequences F: CCTTGC TCTGCCATACCCGT, R: ACACTGGTCCTCTCCTCCCT) and TFF-3 (F: TAACCCTGCTGCTGGTCCTG, R: GTTT GAAGCACCAGGGCACA), and the internal control (GAPDH) was measured by qRT-PCR. Furthermore, gene expressions in Toll-like receptor signaling pathway were determined by real-time PCR array according to the manufacturer's protocol (PAMM-0018ZD, SA Biosciences, Frederick, MD) on CFX96 thermocycler (Bio-Rad, Hercules, CA). Data 


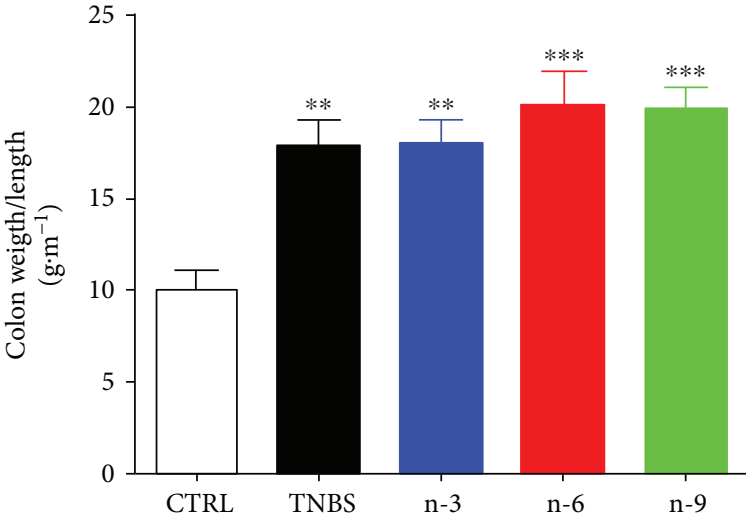

(a)

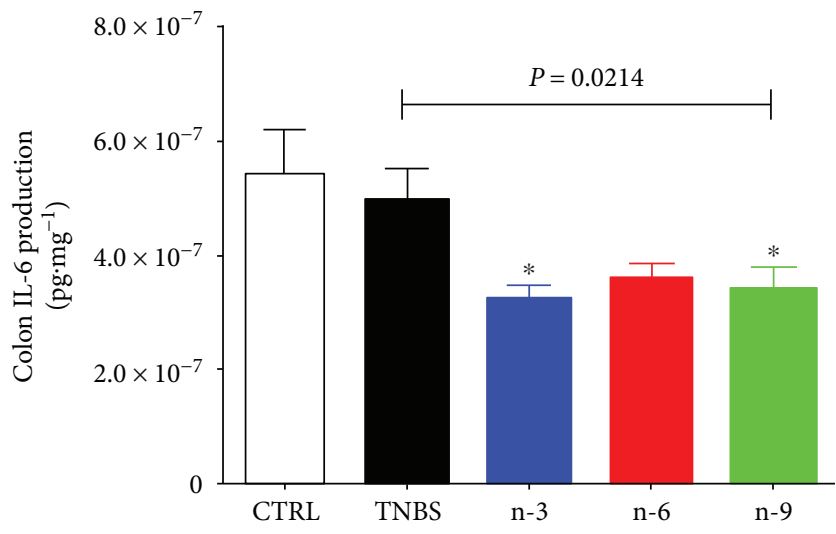

(c)

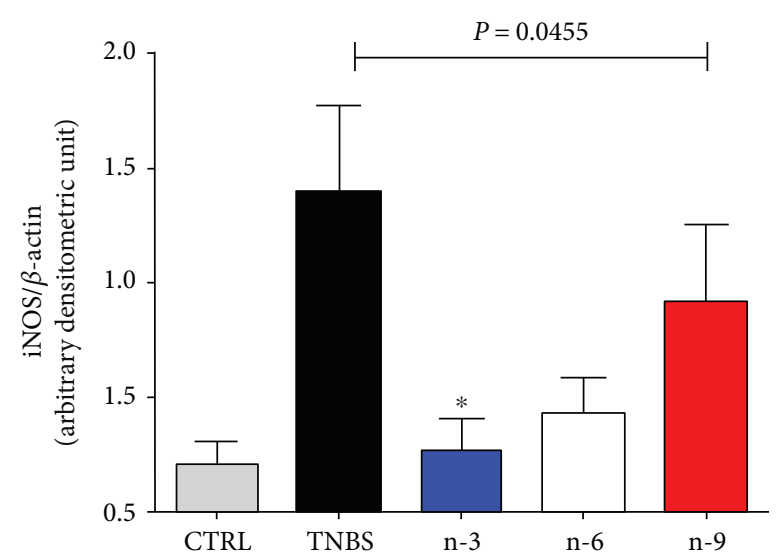

(b)
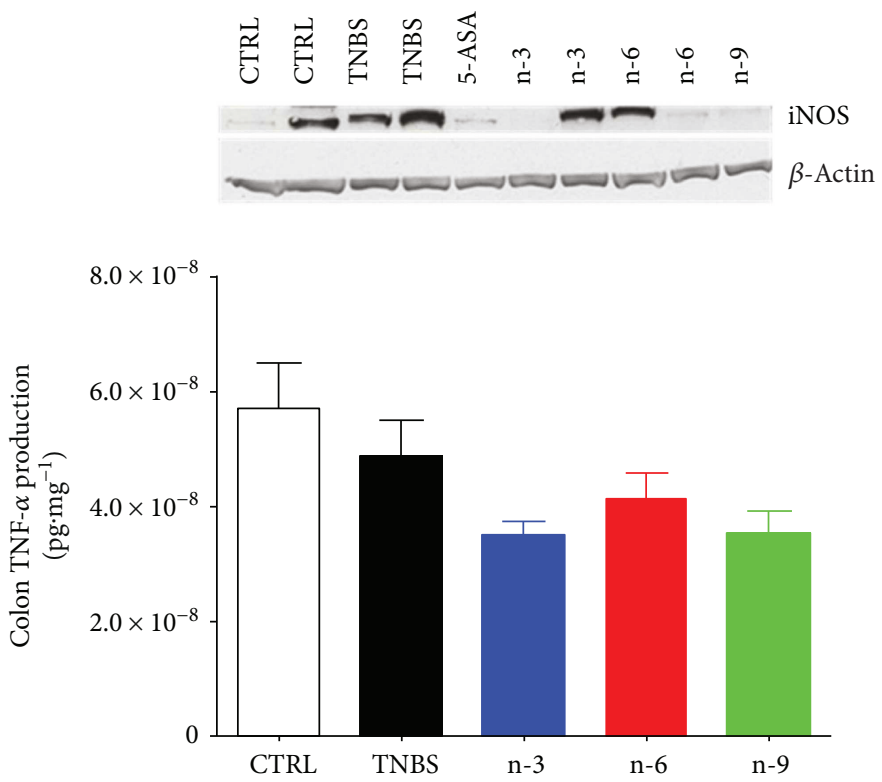

(d)

FIGURE 2: Inflammatory markers in rats receiving diets varying in their unsaturated fatty acid composition for 4 weeks followed by TNBS-induced colitis. (a) Colon/weight length at day 2. (b) Colon iNOS expression with a representative gel at day 2. (c) Colon IL6 and (d) TNF $\alpha$ production. 5-Aminosalicylic acid (5-ASA) is used a positive anti-inflammatory control. Data from colitic rats were compared by 1 -way ANOVA followed by Tukey posttests. $* *$ means $P<0.01$ versus CTRL, $* * *$ means $P<0.001$ versus CTRL, and * means $P<0.05$ versus TNBS.

are expressed in fold regulation. The fold change (fold difference) is calculated by the equation $2(-\Delta \Delta \mathrm{CT})$. For the fold regulation, the software transforms fold change values less than 1 (meaning that the gene is downregulated) by returning the negative inverse.

2.6. Colon Cytokines and LTB4 Production. Concentrations of TNF $\alpha$, IL- $1 \beta$, and LTB4 in the colon homogenates were detected by ELISA (R\&D Systems, Lille, France) following the manufacturer's instructions.

2.7. Proteolytic Pathway Activities. The evaluation of proteolytic activities (caspase-like and chymotrypsin-like) was performed by spectrofluorometric microtiter plate fluorometer (Mithras LB 940, Berthold Technologies) using fluorogenic proteasome substrate in the presence or absence of specific proteasome inhibitors as previously described [27].

2.8. Statistical Analysis. Statistical comparisons were performed using GraphPadPrism 5. Data are expressed as mean \pm SEM. Body weight changes and food intake were analyzed with 2-way ANOVA for repeated measures with Tukey's post hoc tests. All the other variables were analyzed by one-way ANOVA with Bonferroni post hoc test or Kruskal-Wallis test as appropriate. Differences were considered significant at $P<0.05$.

\section{Results}

3.1. TNBS-Induced Colitis Decreased Body Weight. Colitic groups had a lower body weight compared to control rats 


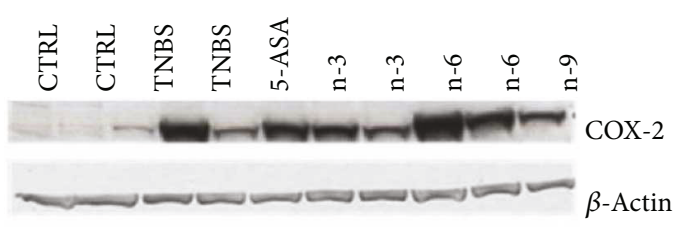

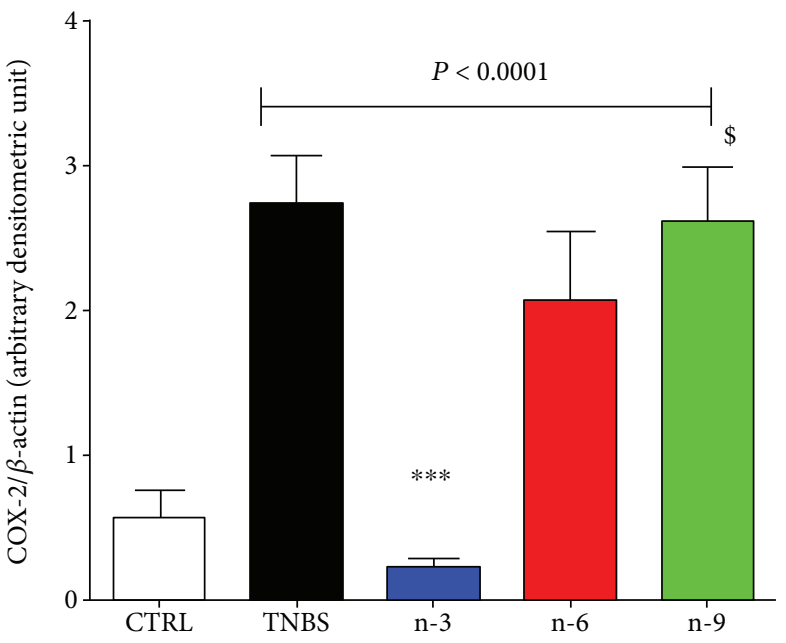

(a)

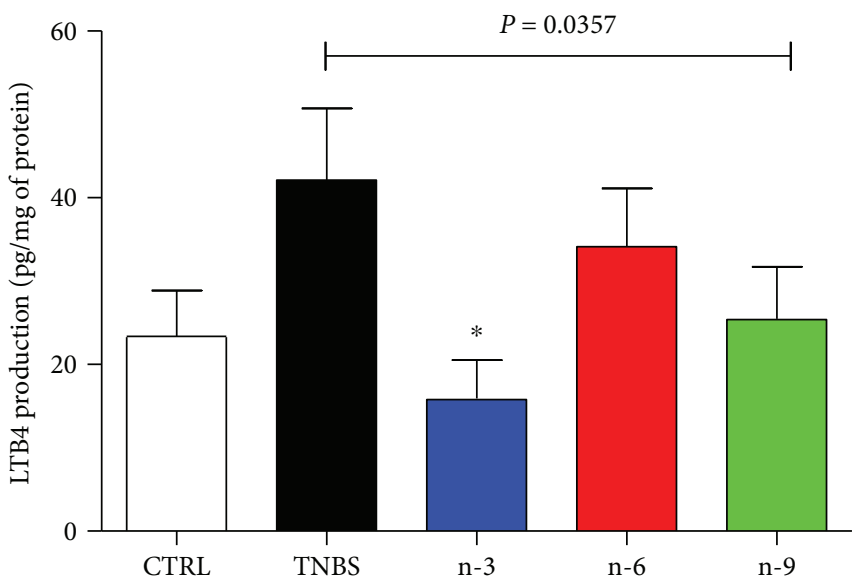

(b)

FIGURE 3: Eicosanoid pathway in rats varying in their unsaturated fatty acid composition for 4 weeks followed by TNBS-induced colitis. (a) Colon cyclooxygenase-2 (COX-2) expression with a representative gel and (b) colon LTB4 production at day 2. 5-Aminosalicylic acid (5-ASA) is used a positive anti-inflammatory control. Data from colitic rats were compared by 1-way ANOVA followed by Tukey posttests. * means $P<0.05$ versus TNBS, *** means $P<0.001$ versus TNBS, and $\$$ means $P<0.05$ versus $\mathrm{n}-3$.

$(P<0.01)$ at day 2 but there is no difference among colitic groups (Figure 1(b)).

3.2. TNBS-Induced Colitis Increased Inflammatory Markers. Colon weight/length ratio was increased in colitic rats compared to control group $(P<0.01$ for TNBS and $\mathrm{n}-3$, $P<0.001$ for n-6 and n-9, Figure 2(a)) without significant differences among colitic groups (Figure 2(a)). Colon iNOS was significantly higher in colitic groups compared to control group $(P=0.0141$, Figure $2(b))$.

3.3. n-3 Diet Decreased Colon Inflammatory Markers. Among colitis groups, n-3 group had a lower colon iNOS compared to TNBS group $(P<0.05$, Figure $2(\mathrm{~b}))$. Colon IL-6 production was significantly lower in n-3 and n- 6 groups compared to TNBS group $(P<0.05$ for both, Figure 2(c)) while colon $\mathrm{TNF} \alpha$ production did not significantly differ among colitis groups (Figure $2(\mathrm{~d})$ ) but tend to decrease in $\mathrm{n}-3$ group compared to TNBS group $(P=0.0617)$. Transcription factors HNF- $4 \alpha$ and PPAR $\gamma$ expressions were not different among groups (data not shown).

3.4. n-3 Diet Decreased COX-2 Expression and LTB4 Production in the Colon. Among colitis groups, n-3 group had a lower colon COX-2 expression compared to TNBS group $(P<0.001$, Figure $3(\mathrm{a}))$. In addition, colon LTB4 production was lower in the $\mathrm{n}-3$ group compared to TNBS group $(P<0.05$, Figure 3(b)).
3.4.1. Gut Barrier Function Was Not Affected by Dietary Treatments. Tight junction proteins claudin-1 and occludin were not different among groups $(P=0.4750$ and $P=0.8553$, resp., Figures $4(\mathrm{a})$ and $4(\mathrm{~b}))$. TFF3 mRNA levels were not different among groups $(P=0.3729$, Figure $4(\mathrm{c}))$. MUC2 mRNA levels were not different among groups (1-way ANOVA, $P=0.0381$, posttests $P>0.05$, Figure $4(\mathrm{~d})$ ).

3.5. Colitis or Dietary PUFA Did Not Modify Proteasome Activity. Chymotrypsin and trypsin-like activities were not different among colitic groups $(P=0.3510$ and $P=0.0651$, resp., data not shown).

3.6. Dietary Modulation of Inflammatory Gene Expression. In colitis groups, n-3 diet upregulated IL-1A, TLR-2, and MA2K3 genes while n-9 diet upregulated TLR-4 genes $(P=0.044, P=0.013$, and $P=0.021$, resp., Table 2$)$. n-6 upregulated HMGB1 $(P=0.042)$ without affecting TLR pathways $(P>0.05$, Table 2$)$.

\section{Discussion}

Numerous experimental studies found anti-inflammatory effects of n-3 PUFA in intestinal inflammation while randomized clinical trials failed to demonstrate efficacy $[2,23]$. We previously hypothesized that the discrepancy between clinical trials and experimental studies could result from the timing of the intervention [23]. In our previous studies in colitis models [16-19], we tested nutritional intervention 


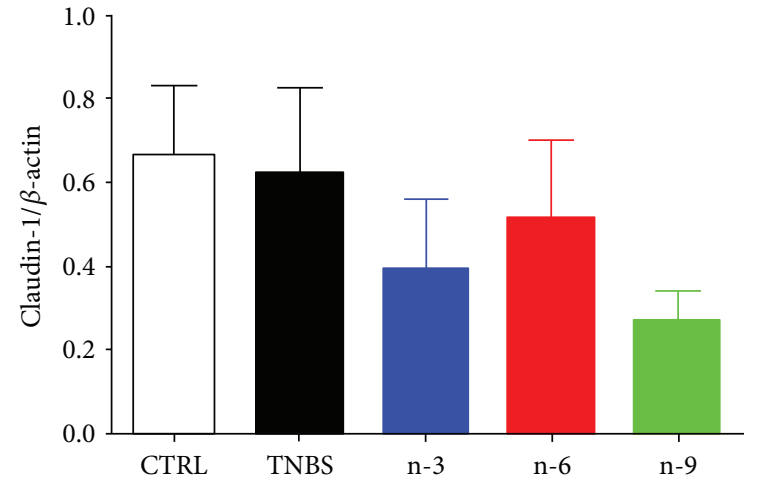

(a)

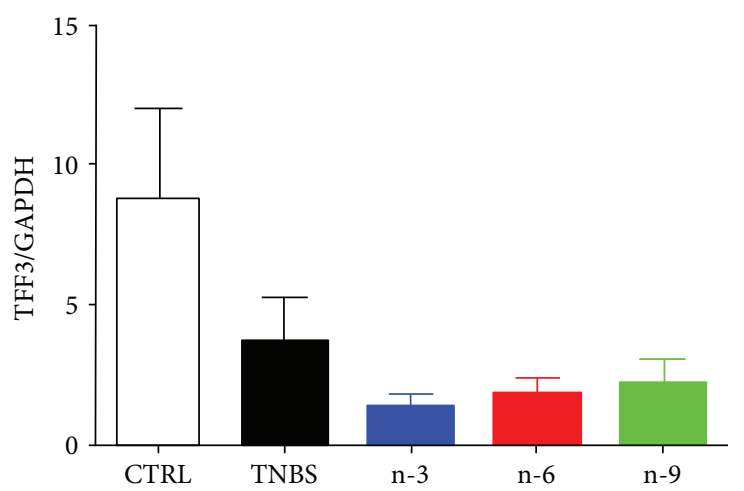

(c)

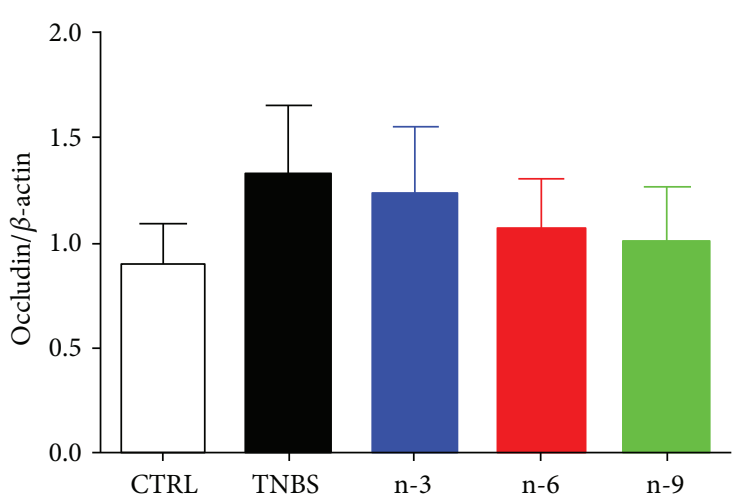

(b)

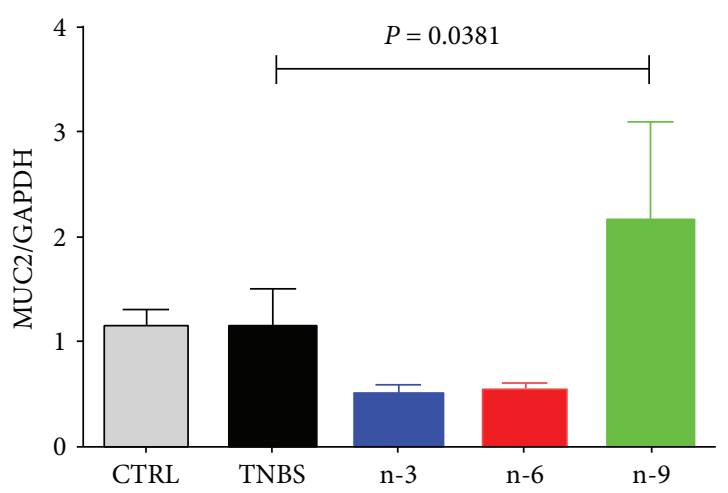

(d)

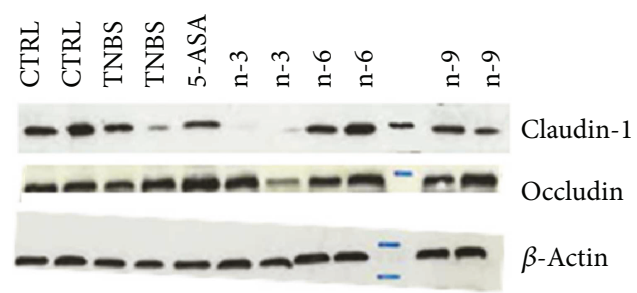

(e)

FIGURE 4: Gut barrier parameters in rats receiving diets varying in their unsaturated fatty acid composition for 4 weeks followed by TNBSinduced colitis. (a) Colon claudin-1 expression, (b) occludin expression, (c) trefoil factor 3 (TFF3) mRNA level, and (d) MUC2 mRNA level at day 2. (e) Representative gel of colon claudin-1 and occludin expression. 5-Aminosalicylic acid (5-ASA) is used a positive anti-inflammatory control. Data from colitic rats were compared by 1-way ANOVA followed by Tukey posttests.

with n-3 PUFA in a curative manner. We now speculated that nutritional intervention with fatty acid should be preventive as reflected in the epidemiological studies. In epidemiological studies, dietary intake of PUFA modifies IBD risk, and identification of their potential mechanisms is now required. To this purpose, we fed rats for four weeks with diets differing in their PUFA composition before the onset of colitis.

In the present study, n-3 diet downregulated colon iNOS expression (Figure 2(a)) in rats with TNBS-induced colitis similar to previous studies performed by us $[16,19]$ or others [21]. Indeed, n-3 PUFA can regulate oxidative stress. Camuesco et al. found that olive oil enriched with fish oil decreased oxidative activity by restoring glutathione concentration and reducing iNOS expression in the colon of rats [21]. Dietary n-3 PUFA exerted anti-inflammatory properties. Indeed, n-3 diet decreased colon COX-2 and colon
LTB4 production (Figure 3). This result is in accordance with our previous studies showing an inhibitory effect of nutritional intervention with n-3 PUFA on COX-2 and LTB4 $[16,19]$. Similarly, it has been shown that antagonizing arachidonic acid-derived eicosanoids reduced inflammation and colitis severity in mice [28]. In addition, alteration of eicosanoids is one of the PUFA main mechanisms [29]. In the present study, n-3 diet also downregulated colon proinflammatory cytokines such as IL-6 (Figure 2(c)) and tend to decrease $\mathrm{TNF} \alpha$ production.

In the present study, IL-1A gene expression was upregulated by n-3 diet (Table 2). This result is in accordance with an in vitro study showing that EPA treatment increased IL-1A secretion in human keratinocytes [30]. In our study, we observed a significant decreased IL-6 production (Figure 2(c)) while IL-6 gene expression did not differ (Table 2). The discrepancy between gene expression and 
TABLE 2: Inflammation pathway PCR array in rats receiving diets varying in their unsaturated fatty acid composition followed by TNBSinduced colitis. Colon RNA from rats receiving diets varying in their unsaturated fatty acid composition before the onset of TNBSinduced colitis. The results were compared to rats receiving TNBS with a control diet. Data in bold are significantly different from TNBS rats.

\begin{tabular}{|c|c|c|c|c|c|c|}
\hline & \multicolumn{2}{|c|}{ n-3 diet } & \multicolumn{2}{|c|}{ n-6 diet } & \multicolumn{2}{|c|}{ n-9 diet } \\
\hline & Fold regulation & $P$ value & Fold regulation & $P$ value & Fold regulation & $P$ value \\
\hline Illa & 2.29 & 0.044 & 1.33 & 0.224 & 1.50 & 0.157 \\
\hline Illb & 1.60 & 0.125 & 1.09 & 0.776 & -1.03 & 0.879 \\
\hline Il12a & -1.64 & 0.113 & -1.12 & 0.532 & -1.41 & 0.226 \\
\hline Il6 & 1.54 & 0.363 & -2.76 & 0.219 & -1.10 & 0.656 \\
\hline TNF & 1.40 & 0.138 & 1.32 & 0.260 & 1.24 & 0.393 \\
\hline Ifng & 1.06 & 0.417 & 2.30 & 0.124 & 1.78 & 0.183 \\
\hline Il10 & 1.49 & 0.378 & -1.36 & 0.793 & 1.16 & 0.776 \\
\hline Il1r1 & 1.39 & 0.205 & 1.26 & 0.446 & 1.11 & 0.864 \\
\hline Hmgb1 & 1.09 & 0.492 & 1.52 & 0.042 & 1.02 & 0.851 \\
\hline Map2k3 & 1.65 & 0.021 & 1.36 & 0.117 & 1.2 & 0.278 \\
\hline $\mathrm{Il} 2$ & -1.27 & 0.904 & 4.23 & 0.258 & 3.13 & 0.275 \\
\hline Clec4e & 1.46 & 0.372 & -2.13 & 0.184 & -1.37 & 0.724 \\
\hline Lta & -1.04 & 0.572 & -2.44 & 0.351 & -1.09 & 0.952 \\
\hline Cd86 & -1.11 & 0.569 & -1.12 & 0.778 & -1.52 & 0.163 \\
\hline Fos & -1.05 & 0.345 & -1.3 & 0.379 & -1.32 & 0.214 \\
\hline Irf1 & -1.32 & 0.451 & 1.08 & 0.988 & 1.02 & 0.693 \\
\hline Jun & -1.37 & 0.340 & 1.09 & 0.762 & -1.34 & 0.158 \\
\hline Tlr1 & 1.23 & 0.419 & -1.05 & 0.803 & 1.06 & 0.654 \\
\hline Tlr2 & 1.73 & 0.013 & 1.18 & 0.320 & 1.16 & 0.371 \\
\hline Tlr3 & -1.38 & 0.320 & 1.25 & 0.659 & 1.11 & 0.846 \\
\hline Tlr4 & 1.49 & 0.140 & 1.34 & 0.251 & 2.01 & 0.005 \\
\hline Tlr5 & -1.09 & 0.805 & 1.18 & 0.815 & 1.49 & 0.758 \\
\hline Tlr6 & 1.26 & 0.381 & 1.02 & 0.855 & 1.05 & 0.960 \\
\hline Tlr7 & -1.37 & 0.397 & -1.44 & 0.207 & -1.29 & 0.299 \\
\hline Tlr9 & -1.24 & 0.802 & -1.18 & 0.877 & -1.47 & 0.285 \\
\hline
\end{tabular}

protein concentration is a frequent finding in the literature. In a previous study, we observed that TNBS administration led to a $60 \%$ increase of $\mathrm{TNF} \alpha$ production, while a 12 -fold increase of gene expression was observed [16]. Studies that have tested correlations between gene expression and protein levels have found that mRNA and protein abundances are differentially expressed, suggesting a frequent posttranscriptional regulation of gene expression [31].

Dietary n-3 PUFA increased TLR2 gene compared to control diet while n-9 diet increased TLR4 gene (Table 2). In the literature, the inhibitory effect of n-3 PUFA on TLR2 is controversial. TLR2 protein expression was downregulated by EPA in mouse adipose stem cells [32] while a study investigating the effect of a range of saturated and unsaturated fats on TLR2 and TLR4 activation found no effect [33]. The investigators of this study did not find any effect on DHA, EPA, or oleic acid to activate TLR2 and TLR4 in HEK-Blue cells [33]. Nevertheless, these fatty acids were able to downregulate cytokine production such as TNF $\alpha$, IL-6, and MCP-1 secretion in human adipose tissue and adipocyte cultures [33]. We studied dietary effects on TLR expression but we did not explore their effects on the intestinal microbiota. It has been demonstrated that fish oil is able to attenuate n-6 PUFA-induced dysbiosis in a colitis model [34].

Dietary n-6 increased gene expression of high-mobility group box 1 (HMGB1, Table 2). An increased of colon HMGB1 by dietary n-6 PUFA was observed in rats with colon cancer [35]. HMGB1 can activate multiple signaling pathways such as TLR but we did not observe any increase in TLR signaling by n-6 diet. Other signaling pathways such as receptor for advanced glycation end products (RAGE) signaling may be involved [36]. Indeed, increased RAGE via dietary n-6 has been reported in experimental colon cancer models [37].

Except for il-1a, MAP2k3, and TLR genes, we observed only modest effect of n-3 diet in inflammatory gene expression. Contrary to other studies, we aimed to evaluate a dietary effect on n-3 PUFA before inflammation genesis while numerous studies are interested in a pharmacological effect of n-3 PUFA in a curative manner [21, 38]. Numerous studies have used long chain n-3 PUFA [39] while the experimental diets used in the present study did not contain any long chain PUFA; these diets cannot directly reproduce a typical omnivore human diet. Route of administration is also a crucial point, and we used diets varying in their unsaturated fatty acid composition while n-3 PUFA are often administered by 
gavage. These experimental design discrepancies may explain our effects on inflammatory gene expression.

Fatty acids are endogenous ligands for HNF- $4 \alpha$ [40], and the role of HNF- $4 \alpha$ in the intestinal inflammatory homeostasis has been demonstrated in mice with the intestinal epithelial deleted HNF-4 $\alpha$ [41]. We hypothesized that dietary PUFA can regulate HNF- 4 but we did not observe any modifications of colon HNF- $4 \alpha$ expression among groups. Similarly, nuclear receptor PPAR $\gamma$ can be activated by PUFA and is a regulator of intestinal inflammation [42, 43], but its expression is not different among groups.

Dietary PUFA did not affect barrier function in our study. We investigated tight junction proteins, MUC2 and TFF3 mRNA levels, and we did not find any significant effect among groups (Figure 4). Some studies found a protective effect of n-3 PUFA on barrier function. Hudert et al. have used transgenic mice carrying the $C$. elegans fat- 1 gene encoding an n-3 fatty acid desaturase that converts n-6 to $\mathrm{n}-3$ fatty acids and they induced DSS colitis in these mice [44]. They found that fat-1 mice were protected from colitis induction compared to wild-type mice with decreased inflammatory markers [44]. They also found that fat-1 mice exhibited an increased production of protective markers such as TFF3 [44]. Fish oil supplementation in rats with TNBSinduced colitis also increased the number of goblet cell with mature mucin granules [38]. Nevertheless, our experimental design is different from these studies. Indeed, we investigated the effect of PUFA at a dietary dose while the previous studies investigated PUFA as immunonutrients.

In the present study, $\mathrm{n}-3$ diet group which showed n-3/n-6 ratio equals to 1 attenuated inflammatory markers in the colon. This preventive approach has been already tested in small clinical trials. In a Japanese study, the efficacy of n-3 diet therapy in IBD patients has been already evaluated [25]. The authors of this study combined a double nutritional approach to achieve a n-3/n-6 ratio of 1 for their patients by dietary advice and nutritional supplementation [25]. Their patients were prohibited from consuming the main source of n-6 PUFA consumption such as vegetable oils or dressings. They also provided a n-3 PUFA food exchange table to privilege and $n-3$ supplementation [25]. The authors of this study found a higher n-3/n- 6 ratio in the remission group [25]. In a Norwegian study, they evaluated the effect of $600 \mathrm{~g}$ of salmon consumption per week for 8 weeks in 12 active UC patients and they found decreased clinical inflammatory index [45]. A proof of concept study is now required to evaluate $n-3$ PUFA in a preventive manner. As we cannot directly target IBD physiopathology with a nutritional therapy before the IBD diagnosis, we may first evaluate $n-3$ therapy in $\mathrm{CD}$ postoperative patients. Indeed, postoperative phase is considered as a perfect window to evaluate predisposing factors to IBD recurrence.

Similarly, in a recent epidemiological study, women with a prudent diet (characterized by greater intake of fruits, vegetables, and fish) had a lower $C D$ risk [15]. In addition, greater intake of fish $(P$ trend $=0.01)$ has been specifically associated with lower risk of $\mathrm{CD}$ [15].

In conclusion, prudent diet with a high n-3/n-6 ratio may contribute to partially limit colitis genesis. Further research will be mandatory to determine mechanisms underlying dietary effects to better define dietetic advice with a scientific rationale.

\section{Conflicts of Interest}

The authors declare that they have no conflicts of interest.

\section{References}

[1] D. C. Baumgart and S. R. Carding, "Inflammatory bowel disease: cause and immunobiology," The Lancet, vol. 369, no. 9573, pp. 1627-1640, 2007.

[2] J. D. Lewis and M. T. Abreu, "Diet as a trigger or therapy for inflammatory bowel diseases," Gastroenterology, vol. 152, pp. 398-414.e6, 2016.

[3] C. Zallot, D. Quilliot, J. B. Chevaux et al., "Dietary beliefs and behavior among inflammatory bowel disease patients," Inflammatory Bowel Diseases, vol. 19, no. 1, pp. 66-72, 2013.

[4] F. Bergeron, M. Bouin, L. D'Aoust, M. Lemoyne, and N. Presse, "Food avoidance in patients with inflammatory bowel disease: what, when and who?," Clinical Nutrition, 2017.

[5] R. Shoda, K. Matsueda, S. Yamato, and N. Umeda, "Epidemiologic analysis of Crohn disease in Japan: increased dietary intake of n-6 polyunsaturated fatty acids and animal protein relates to the increased incidence of Crohn disease in Japan," The American Journal of Clinical Nutrition, vol. 63, no. 5, pp. 741-745, 1996.

[6] P. Jantchou, S. Morois, F. Clavel-Chapelon, M. C. BoutronRuault, and F. Carbonnel, "Animal protein intake and risk of inflammatory bowel disease: the E3N prospective study," The American Journal of Gastroenterology, vol. 105, no. 10, pp. 2195-2201, 2010.

[7] J. K. Hou, B. Abraham, and H. El-Serag, "Dietary intake and risk of developing inflammatory bowel disease: a systematic review of the literature," The American Journal of Gastroenterology, vol. 106, no. 4, pp. 563-573, 2011.

[8] L. Tasson, C. Canova, M. G. Vettorato, E. Savarino, and R. Zanotti, "Influence of diet on the course of inflammatory bowel disease," Digestive Diseases and Sciences, vol. 62, no. 8, pp. 2087-2094, 2017.

[9] M. Martinez-Medina, J. Denizot, N. Dreux et al., "Western diet induces dysbiosis with increased E coli in CEABAC10 mice, alters host barrier function favouring AIEC colonisation," Gut, vol. 63, no. 1, pp. 116-124, 2014

[10] T. L. Blasbalg, J. R. Hibbeln, C. E. Ramsden, S. F. Majchrzak, and R. R. Rawlings, "Changes in consumption of omega-3 and omega- 6 fatty acids in the United States during the 20th century," The American Journal of Clinical Nutrition, vol. 93, no. 5, pp. 950-962, 2011.

[11] IBD in EPIC Study Investigators, A. Tjonneland, K. Overvad et al., "Linoleic acid, a dietary n- 6 polyunsaturated fatty acid, and the aetiology of ulcerative colitis: a nested case-control study within a European prospective cohort study," Gut, vol. 58, no. 12, pp. 1606-1611, 2009.

[12] S. John, R. Luben, S. S. Shrestha, A. Welch, K. T. Khaw, and A. R. Hart, "Dietary n-3 polyunsaturated fatty acids and the aetiology of ulcerative colitis: a UK prospective cohort study," European Journal of Gastroenterology \& Hepatology, vol. 22, no. 5, pp. 602-606, 2010. 
[13] S. Rashvand, M. H. Somi, B. Rashidkhani, and A. Hekmatdoost, "Dietary fatty acid intakes are related to the risk of ulcerative colitis: a case-control study," International Journal of Colorectal Disease, vol. 30, no. 9, pp. 1255-1260, 2015.

[14] P. S. A. de Silva, R. Luben, S. S. Shrestha, K. T. Khaw, and A. R. Hart, "Dietary arachidonic and oleic acid intake in ulcerative colitis etiology: a prospective cohort study using 7-day food diaries," European Journal of Gastroenterology \& Hepatology, vol. 26, no. 1, pp. 11-18, 2014.

[15] A. N. Ananthakrishnan, H. Khalili, M. Song et al., "High school diet and risk of Crohn's disease and ulcerative colitis," Inflammatory Bowel Diseases, vol. 21, no. 10, pp. 2311-2319, 2015.

[16] A. Hassan, A. Ibrahim, K. Mbodji et al., "An $\alpha$-linolenic acid-rich formula reduces oxidative stress and inflammation by regulating NF- $\kappa \mathrm{B}$ in rats with TNBS-induced colitis," The Journal of Nutrition, vol. 140, no. 10, pp. 1714-1721, 2010.

[17] A. Ibrahim, M. Aziz, A. Hassan et al., "Dietary $\alpha$-linolenic acid-rich formula reduces adhesion molecules in rats with experimental colitis," Nutrition, vol. 28, no. 7-8, pp. 799-802, 2012.

[18] A. Ibrahim, K. Mbodji, A. Hassan et al., "Anti-inflammatory and anti-angiogenic effect of long chain n-3 polyunsaturated fatty acids in intestinal microvascular endothelium," Clinical Nutrition, vol. 30, no. 5, pp. 678-687, 2011.

[19] K. Mbodji, C. Charpentier, C. Guérin et al., "Adjunct therapy of n-3 fatty acids to 5-ASA ameliorates inflammatory score and decreases NF- $\kappa \mathrm{B}$ in rats with TNBS-induced colitis," The Journal of Nutritional Biochemistry, vol. 24, no. 4, pp. 700705, 2013.

[20] D. Camuesco, M. Comalada, A. Concha et al., "Intestinal antiinflammatory activity of combined quercitrin and dietary olive oil supplemented with fish oil, rich in EPA and DHA (n-3) polyunsaturated fatty acids, in rats with DSS-induced colitis," Clinical Nutrition, vol. 25, no. 3, pp. 466-476, 2006.

[21] D. Camuesco, J. Gálvez, A. Nieto et al., "Dietary olive oil supplemented with fish oil, rich in EPA and DHA (n-3) polyunsaturated fatty acids, attenuates colonic inflammation in rats with DSS-induced colitis," The Journal of Nutrition, vol. 135, no. 4, pp. 687-694, 2005.

[22] B. G. Feagan, W. J. Sandborn, U. Mittmann et al., “Omega-3 free fatty acids for the maintenance of remission in Crohn disease: the EPIC randomized controlled trials," JAMA, vol. 299, no. 14, pp. 1690-1697, 2008.

[23] R. Marion-Letellier, G. Savoye, P. L. Beck, R. Panaccione, and S. Ghosh, "Polyunsaturated fatty acids in inflammatory bowel diseases: a reappraisal of effects and therapeutic approaches," Inflammatory Bowel Diseases, vol. 19, no. 3, pp. 650-661, 2013.

[24] H. Blanchard, F. Pédrono, N. Boulier-Monthéan, D. Catheline, V. Rioux, and P. Legrand, "Comparative effects of wellbalanced diets enriched in $\alpha$-linolenic or linoleic acids on LC-PUFA metabolism in rat tissues," Prostaglandins, Leukotrienes and Essential Fatty Acids (PLEFA), vol. 88, no. 5, pp. 383-389, 2013.

[25] K. Uchiyama, M. Nakamura, S. Odahara et al., "N-3 polyunsaturated fatty acid diet therapy for patients with inflammatory bowel disease," Inflammatory Bowel Diseases, vol. 16, no. 10, pp. 1696-1707, 2010.

[26] M. de Lorgeril, P. Salen, J. L. Martin, I. Monjaud, J. Delaye, and N. Mamelle, "Mediterranean diet, traditional risk factors, and the rate of cardiovascular complications after myocardial infarction: final report of the Lyon Diet Heart Study," Circulation, vol. 99, no. 6, pp. 779-785, 1999.

[27] J. Bertrand, R. Marion-Letellier, S. Azhar et al., "Glutamine enema regulates colonic ubiquitinated proteins but not proteasome activities during TNBS-induced colitis leading to increased mitochondrial activity," Proteomics, vol. 15, no. 13, pp. 2198-2210, 2015.

[28] J. M. Monk, H. F. Turk, Y.-Y. Fan et al., "Antagonizing arachidonic acid-derived eicosanoids reduces inflammatory Th17 and Th1 cell-mediated inflammation and colitis severity," Mediators of Inflammation, vol. 2014, Article ID 917149, 14 pages, 2014.

[29] R. Marion-Letellier, G. Savoye, and S. Ghosh, "Polyunsaturated fatty acids and inflammation," IUBMB Life, vol. 67, no. 9, pp. 659-667, 2015.

[30] A. Pupe, P. De Haes, L. Rhodes et al., "Eicosapentaenoic acid, a n-3 polyunsaturated fatty acid differentially modulates TNF- $\alpha$, IL- $1 \alpha$, IL- 6 and PGE 2 expression in UVB-irradiated human keratinocytes," Journal of Investigative Dermatology, vol. 118, no. 4, pp. 692-698, 2002.

[31] L. Anderson and J. Seilhamer, "A comparison of selected mRNA and protein abundances in human liver," Electrophoresis, vol. 18, no. 3-4, pp. 533-537, 1997.

[32] H. W. Hsueh, Z. Zhou, J. Whelan et al., "Stearidonic and eicosapentaenoic acids inhibit interleukin-6 expression in $o b / o b$ mouse adipose stem cells via toll-like receptor-2-mediated pathways," The Journal of Nutrition, vol. 141, no. 7, pp. 1260-1266, 2011.

[33] R. K. Murumalla, M. K. Gunasekaran, J. K. Padhan et al., "Fatty acids do not pay the toll: effect of SFA and PUFA on human adipose tissue and mature adipocytes inflammation," Lipids in Health and Disease, vol. 11, no. 1, p. 175, 2012.

[34] S. Ghosh, D. DeCoffe, K. Brown et al., "Fish oil attenuates omega-6 polyunsaturated fatty acid-induced dysbiosis and infectious colitis but impairs LPS dephosphorylation activity causing sepsis," PLoS One, vol. 8, no. 2, article e55468, 2013.

[35] H. Ohmori, Y. Luo, K. Fujii et al., "Dietary linoleic acid and glucose enhances azoxymethane-induced colon cancer and metastases via the expression of high-mobility group box 1 ," Pathobiology, vol. 77, no. 4, pp. 210-217, 2010.

[36] H. E. Harris, U. Andersson, and D. S. Pisetsky, "HMGB1: a multifunctional alarmin driving autoimmune and inflammatory disease," Nature Reviews Rheumatology, vol. 8, no. 4, pp. 195-202, 2012.

[37] T. Shimomoto, Y. Luo, H. Ohmori et al., "Advanced glycation end products (AGE) induce the receptor for AGE in the colonic mucosa of azoxymethane-injected Fischer 344 rats fed with a high-linoleic acid and high-glucose diet," Journal of Gastroenterology, vol. 47, no. 10, pp. 1073-1083, 2012.

[38] N. Nieto, M. I. Torres, A. Rios, and A. Gil, "Dietary polyunsaturated fatty acids improve histological and biochemical alterations in rats with experimental ulcerative colitis," The Journal of Nutrition, vol. 132, no. 1, pp. 11-19, 2002.

[39] J. Bassaganya-Riera and R. Hontecillas, "CLA and n-3 PUFA differentially modulate clinical activity and colonic PPAR-responsive gene expression in a pig model of experimental IBD," Clinical Nutrition, vol. 25, no. 3, pp. 454-465, 2006.

[40] S. Dhe-Paganon, K. Duda, M. Iwamoto, Y. I. Chi, and S. E. Shoelson, "Crystal structure of the HNF $4 \alpha$ ligand binding 
domain in complex with endogenous fatty acid ligand," Journal of Biological Chemistry, vol. 277, no. 41, pp. 3797337976, 2002.

[41] M. Darsigny, J.-P. Babeu, A.-A. Dupuis et al., "Loss of hepatocyte-nuclear-factor- $4 \alpha$ affects colonic ion transport and causes chronic inflammation resembling inflammatory bowel disease in mice," PLoS One, vol. 4, no. 10, article e7609, 2009.

[42] R. Marion-Letellier, M. Butler, P. Dechelotte, R. J. Playford, and S. Ghosh, "Comparison of cytokine modulation by natural peroxisome proliferator-activated receptor $\gamma$ ligands with synthetic ligands in intestinal-like Caco-2 cells and human dendritic cells-potential for dietary modulation of peroxisome proliferator-activated receptor $\gamma$ in intestinal inflammation," The American Journal of Clinical Nutrition, vol. 87, no. 4, pp. 939-948, 2008.

[43] R. Marion-Letellier, P. Dechelotte, M. Iacucci, and S. Ghosh, "Dietary modulation of peroxisome proliferator-activated receptor gamma," Gut, vol. 58, no. 4, pp. 586-593, 2009.

[44] C. A. Hudert, K. H. Weylandt, Y. Lu et al., "Transgenic mice rich in endogenous omega-3 fatty acids are protected from colitis," Proceedings of the National Academy of Sciences of the United States of America, vol. 103, no. 30, pp. 11276-11281, 2006.

[45] T. Grimstad, R. K. Berge, P. Bohov et al., "Salmon diet in patients with active ulcerative colitis reduced the simple clinical colitis activity index and increased the antiinflammatory fatty acid index - a pilot study," Scandinavian Journal of Clinical and Laboratory Investigation, vol. 71, no. 1, pp. 68-73, 2011. 


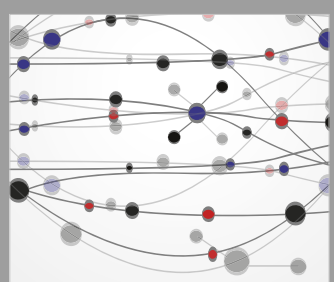

The Scientific World Journal
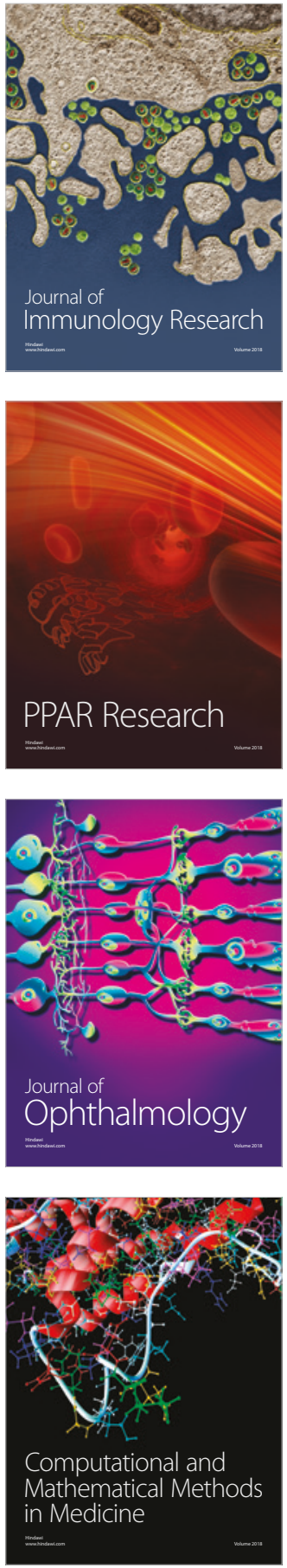

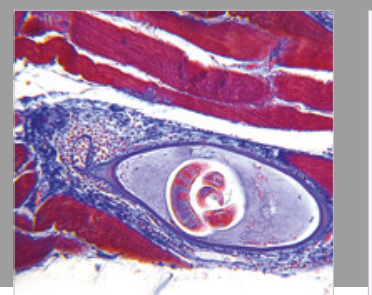

Gastroenterology Research and Practice

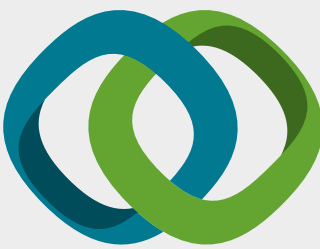

\section{Hindawi}

Submit your manuscripts at

www.hindawi.com
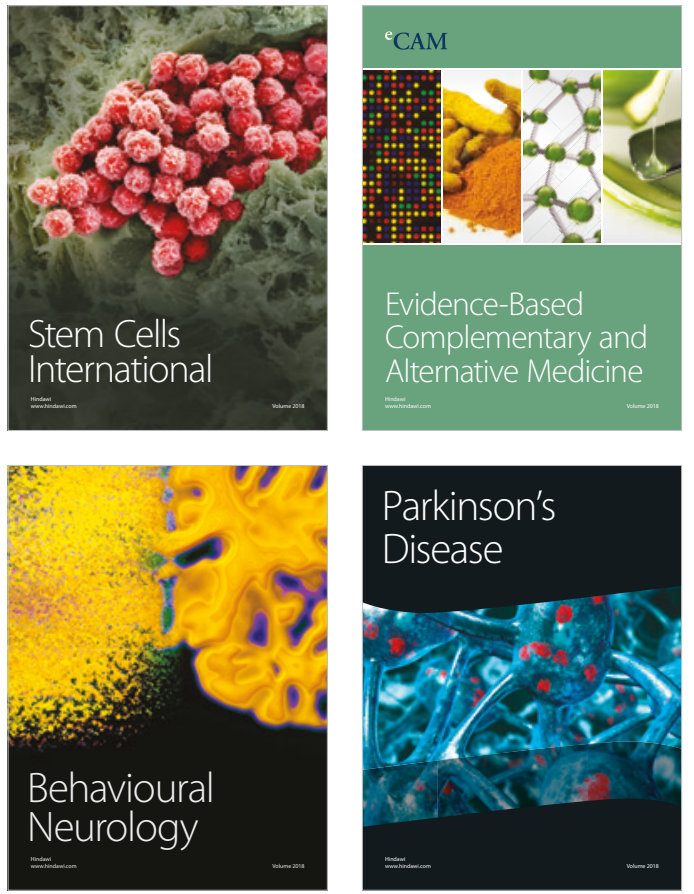

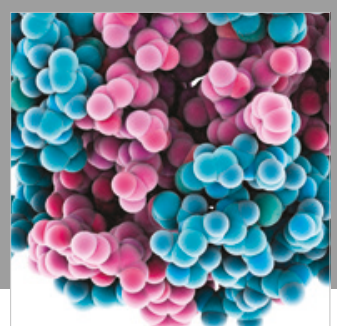

ournal of

Diabetes Research

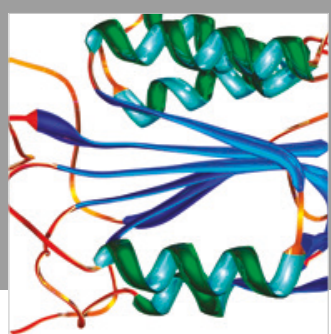

Disease Markers
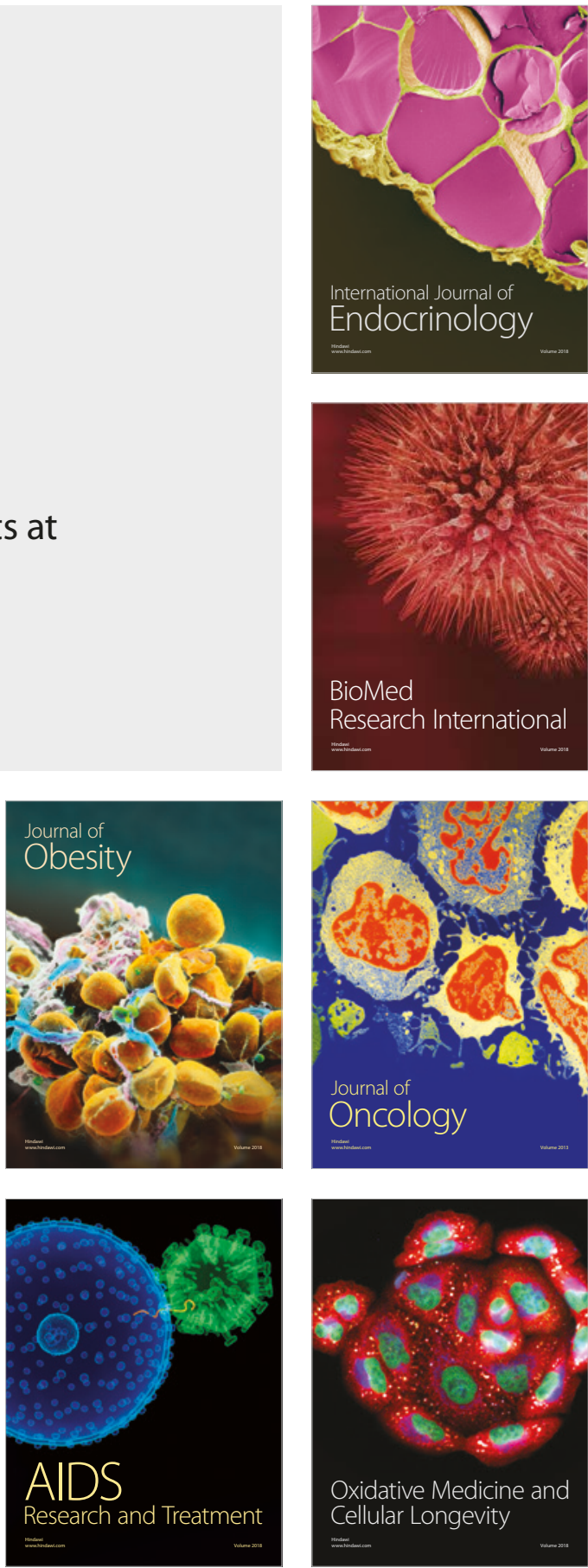